\title{
The proton size puzzle: experiment vs theory.
}

\author{
A. E. Dorokhov ${ }^{1, *}$, A. P. Martynenko ${ }^{2, * *}$, F. A. Martynenko ${ }^{2, * * *}$, and A. E. Radzhabov ${ }^{3,4, * * * *}$ \\ ${ }^{1}$ Bogoliubov Laboratory of Theoretical Physics, JINR, 141980 Dubna, Russia \\ ${ }^{2}$ Samara University, 443086, Samara, Russia \\ ${ }^{3}$ Matrosov Institute for System Dynamics and Control Theory SB RAS, 664033, Irkutsk, Russia \\ ${ }^{4}$ Institute of Modern Physics of Chinese Academy of Sciences, 730000, Lanzhou, China
}

\begin{abstract}
.
Current status of the proton size puzzle from experimental and theoretical points of view is briefly discussed. The interest to these studies is primarily related to experiments conducted by the CREMA collaboration (Charge Radius Experiments with Muonic Atoms) with muonic hydrogen and deuterium by methods of laser spectroscopy. As a result a more accurate value of the proton charge radius was found to be $r_{p}=0.84184(67) \mathrm{fm}$, which is different from the value recommended by CODATA for $7 \sigma$.

In the second part we discuss recent calculations of the contribution of light pseudoscalar (PS) and axial-vector (AV) mesons to the interaction operator of a muon and a proton in muonic hydrogen atom, with the coupling of mesons to the muon being via two-photon intermediate state. Numerical estimates of the contributions to the hyperfine structure of the spectrum of the $\mathrm{S}$ and $\mathrm{P}$ levels are presented. It is shown that such contribution to the hyperfine splitting in muonic hydrogen is rather important for a comparison with precise experimental data.
\end{abstract}

\section{Proton radius puzzle}

Precise investigation of the Lamb shift (LS) and hyperfine structure (HFS) of light muonic atoms is a fundamental problem for testing the Standard model (SM), in particular the quantum electrodynamics calculations, establishing the exact values of SM parameters, such as the Rydberg constant, detailed study of the proton structure, and searching for effects of new physics. Recently, the CREMA (Charge Radius Experiments with Muonic Atoms) Collaboration from PSI by using the laser spectroscopy method measured with unprecedented accuracy the transition frequencies between the $2 \mathrm{P}$ and $2 \mathrm{~S}$ states in muonic hydrogen $(\mu p)$ [1-3]

$$
\begin{aligned}
v_{t}\left(2 \mathrm{P}_{3 / 2}^{F=2}-2 \mathrm{~S}_{1 / 2}^{F=1}\right)=49881.35(65) \mathrm{GHz}, \quad h v_{t}=\Delta E\left(2 \mathrm{P}_{3 / 2}^{F=2}-2 \mathrm{~S}_{1 / 2}^{F=1}\right) \\
v_{s}\left(2 \mathrm{P}_{3 / 2}^{F=1}-2 \mathrm{~S}_{1 / 2}^{F=0}\right)=54611.16(1.05) \mathrm{GHz}, \quad h v_{s}=\Delta E\left(2 \mathrm{P}_{3 / 2}^{F=1}-2 \mathrm{~S}_{1 / 2}^{F=0}\right) .
\end{aligned}
$$

\footnotetext{
*e-mail: dorokhov@ theor.jinr.ru

**e-mail: a.p.martynenko@samsu.ru

***e-mail: f.a.martynenko@gmail.com

****e-mail: aradzh@icc.ru
} 
From these two transition measurements, both the Lamb shift $\Delta E_{L S}$ and the 2S-HFS $\Delta E_{H F S}$ was determined independently with the result [2]

$$
\begin{aligned}
& \Delta E_{L S}^{\exp }\left(2 \mathrm{P}_{1 / 2}-2 \mathrm{~S}_{1 / 2}\right)=202.3706(23) \mathrm{meV}, \\
& \Delta E_{H F S}^{\exp }\left(2 \mathrm{~S}_{1 / 2}^{F=1}-2 \mathrm{~S}_{1 / 2}^{F=0}\right)=22.8089(51) \mathrm{meV} .
\end{aligned}
$$

From theory side these quantities calculated in the frame work of bound-state QED are expressed in terms of the charge $\mathrm{r}_{E}^{2}$ and Zemach $\mathrm{r}_{Z}$ radii of the proton as [3]

$$
\begin{aligned}
& \Delta E_{\mathrm{LS}}^{\mathrm{th}}=206.0668(25)-5.2275(10) \mathrm{r}_{E}^{2} \mathrm{meV}, \\
& \Delta E_{\mathrm{HFS}}^{\mathrm{th}}=22.9843(30)-0.1621(10) \mathrm{r}_{Z} \mathrm{meV},
\end{aligned}
$$

where the charge RMS radius $r_{E}$ is defined via the normalized proton charge distribution $\rho_{\mathrm{E}}$ and the Zemach radius is correlated with the proton magnetic moment distribution $\rho_{\mathrm{M}}$ as

$$
\mathrm{r}_{\mathrm{E}}^{2}=\int d^{3} r r^{2} \rho_{\mathrm{E}}(r), \quad \mathrm{r}_{Z}=\int d^{3} r \int d^{3} r^{\prime} r^{\prime} \rho_{\mathrm{E}}(r) \rho_{\mathrm{M}}\left(r-r^{\prime}\right) .
$$

The first term on the right side of (5) accounts for radiative, relativistic, and recoil effects, while the first term on the right side of (6) is the Fermi energy arising from the interaction between the muon and the proton magnetic moment and different corrections to it. It is also important, that like for the problem of the anomalous magnetic moments of leptons $[4,5]$, the coefficients in front of the radius terms in (5), (6) are much stronger enhanced for the muonic system relative to the electronic system, and thus much more sensitive in extraction of the radii parameters from the experimental data.

The comparison of (5),(6) with (3),(4) provides [2, 3]

$$
\mathrm{r}_{\mathrm{E}}^{\mathrm{CREMA}}=0.84087(39) \mathrm{fm}, \quad \mathrm{r}_{\mathrm{Z}}^{\text {CREMA }}=1.082(37) \mathrm{fm} .
$$

This should be compared with the values recommended by CODATA [7] and based on the electron-proton scattering and electronic hydrogen spectroscopy

$$
\mathrm{r}_{\mathrm{E}}^{\text {CODATA }}=0.8751(61) \mathrm{fm} .
$$

Thus we see two basic results of the laser spectroscopy experiment for $\mu p$. First, from the $\mu p$ spectroscopy the value of $\mathrm{r}_{\mathrm{E}}$ is determined with precision 10 times higher than from electronic data. Second, and more striking, that there is the large discrepancy between $r_{E}^{C R E M A}$ and $\mathrm{r}_{\mathrm{E}}^{\mathrm{CODATA}}$ at the level of $5.6 \sigma$ (or the muonic hydrogen value is $4 \%$ smaller than the CODATA value). This is so-called the proton size puzzle. Later on, the similar problem was detected for the deuteron radius [6].

At present, several experimental groups plan to measure HFS of various muonic atoms with more high precision [8-10]. This will make it possible to better understand the existing "puzzle" of the proton charge radius, to check the Standard Model with greater accuracy and, possibly, to reveal the source of previously unaccounted interactions between the particles forming the bound state in QED.

More recent study [11] of the $(2 S-4 P)$ transition in the electronic hydrogen atom yielded a new value of the proton charge radius, $r_{p}=0.8327(87) \mathrm{fm}$, which is quite consistent with the CREMA data. But new measurement of the transition frequency $(1 S-3 S)$ transition in the electronic hydrogen atom [12] gave the value of the proton charge radius, $r_{p}=0.877(13) \mathrm{fm}$, which completely agrees with the CODATA value. One of the ways of overcoming the controversial situation arises from a deeper theoretical analysis of the fine and hyperfine structure of muonic atom spectrum, with the verification of previously calculated contributions and the 
more accurate construction of the particle interaction operator in quantum field theory, the calculation of new corrections whose value for muonic atoms can increase substantially in comparison with electronic atoms. The expected results will allow to get also a new very important information about the forces which are responsible for the structure of atoms.

\section{Light meson exchange contributions to HFS of $\mu p$}

From the theory side it is urgently needed to study the possible effects of exchanges between muon and proton which can contribute to HFS of $\mu p$. Some of such effects was considered in recent papers [13-18]. Below, we discuss the effects of exchanges between muon and proton which can contribute to HFS of $\mu p$ coming from the light pseudoscalar (PS) and axial-vector (AV) meson exchanges between muon and proton induced by meson coupling to muon through two photons (see Fig. 1Left).

The leading contribution to HFS of $\mu p$ is coming from one-photon exchange and has the following form [19-21]:

$$
\begin{aligned}
\Delta V_{B}^{h f s}= & \frac{8 \pi \alpha \mu_{p}}{3 m_{\mu} m_{p}}\left(\mathbf{S}_{p} \mathbf{S}_{\mu}\right) \delta(\mathbf{r})-\frac{\alpha \mu_{p}\left(1+a_{\mu}\right)}{m_{\mu} m_{p} r^{3}}\left[\left(\mathbf{S}_{p} \mathbf{S}_{\mu}\right)-3\left(\mathbf{S}_{p} \mathbf{n}\right)\left(\mathbf{S}_{p} \mathbf{n}\right)\right] \\
& +\frac{\alpha \mu_{p}}{m_{\mu} m_{p} r^{3}}\left[1+\frac{m_{\mu}}{m_{p}}-\frac{m_{\mu}}{2 m_{p} \mu_{p}}\right]\left(\mathbf{L} \mathbf{S}_{p}\right)
\end{aligned}
$$

where $m_{\mu}, \mathbf{S}_{\mu}$ and $m_{p}, \mathbf{S}_{p}$ are masses and spins of muon and proton, correspondingly, $\mu_{p}$ is the proton magnetic moment. The potential (10) gives the main contribution of order $\alpha^{4}$ to the HFS of muonic atom. Precise calculation of the HFS of the spectrum, which is necessary for comparison with modern experimental data, requires the consideration of various corrections accounting for the vacuum polarization, nuclear structure and recoil, and relativistic effects [19, 22-26].

We calculate further the contribution to HFS coming from the pion ${ }^{1}$ and axial-vector $f_{1}(1285), a_{1}(1260)$ and $f_{1}(1420)$ meson exchanges shown in Fig. 1(Left). The effective vertices of the interaction of the PS and AV mesons and virtual photons can be expressed in terms of the transition form factors as follows:

$$
\begin{aligned}
& V^{\mu \nu}\left(k_{1}, k_{2}\right)=i \varepsilon^{\mu \nu \alpha \beta} k_{1 \alpha} k_{2 \beta} \frac{\alpha}{\pi F_{\pi}} F_{\pi^{0} \gamma^{*} \gamma^{*}}\left(k_{1}^{2}, k_{2}^{2}\right), \\
& T^{\mu \nu \alpha}\left(k_{1}, k_{2}\right)=8 \pi i \alpha \varepsilon_{\mu \nu \alpha \tau}\left(k_{1}^{\tau} k_{2}^{2}-k_{2}^{\tau} k_{1}^{2}\right) F_{A V \gamma^{*} \gamma^{*}}^{(0)}\left(k_{1}^{2}, k_{2}^{2}\right),
\end{aligned}
$$

where $k_{1}, k_{2}$ are four-momenta of virtual photons. For small values of the relative momenta of particles in the initial and final states and small value of transfer momentum $t$ between muon and proton, one has $k_{2} \approx-k_{1}, k_{1}^{2} \approx k_{2}^{2} \approx k^{2}$.

The final result for the HFS potential is equal to

$$
\begin{aligned}
& \Delta V_{P S}^{h f s}(\mathbf{t})=\frac{\alpha^{2}}{6 \pi^{2}} \frac{g_{p}}{m_{p} F_{\pi}} \frac{\mathbf{t}^{2}}{\mathbf{t}^{2}+m_{\pi}^{2}} \mathcal{A}\left(\mathbf{t}^{2}\right), \\
& \Delta V_{A V}^{h f s}(\mathbf{t})=-\frac{32 \alpha^{2} g_{A V P P} F_{A V \gamma \gamma}(0,0)}{3 \pi^{2}\left(\mathbf{t}^{2}+M_{A}^{2}\right)} I\left(\frac{m_{\mu}}{\Lambda_{A}}\right) .
\end{aligned}
$$

where

$$
\begin{aligned}
\mathcal{A}\left(\mathbf{t}^{2}\right) & =\frac{2}{\pi^{2} \mathbf{t}^{2}} \int \frac{i d^{4} k\left[t^{2} k^{2}-(t k)^{2}\right]}{k^{2}(k-t)^{2}\left(k^{2}-2 m_{\mu} k_{0}\right)} F_{P S \gamma^{*} \gamma^{*}}\left(k^{2}, k^{2}\right), \\
I\left(\frac{m_{\mu}}{\Lambda_{A}}\right) & =\int \frac{i d^{4} k\left(2 k^{2}+k_{0}^{2}\right)}{k^{2}\left(k^{2}-2 m_{\mu} k_{0}\right)} F_{A V \gamma^{*} \gamma^{*}}\left(k^{2}, k^{2}\right) .
\end{aligned}
$$

\footnotetext{
${ }^{1}$ The contribution of the $\eta$ and $\eta^{\prime}$ mesons is negligible [13]
} 

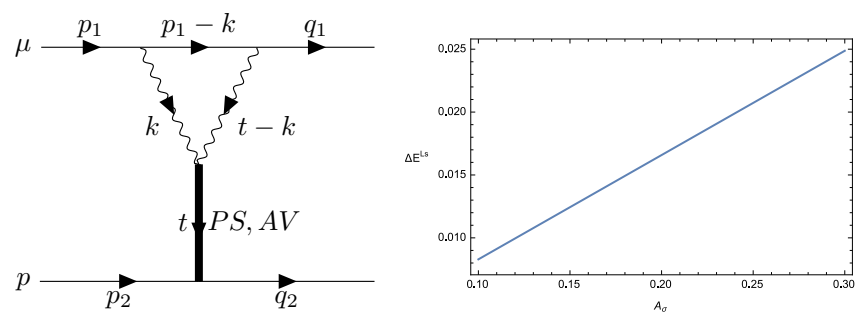

Figure 1. (Left) Muon-proton interaction induced by mesonic exchange; (Right) The contribution of scalar $\sigma$-meson exchange to the Lamb shift of (2P-2S) in meV as function of parameter $A_{\sigma}$ in $\mathrm{GeV}^{-2}$.

The integral $\mathcal{A}$ in (15) is well studied in connection to the problem of interpretation [27,28] of the $\mathrm{KTeV}$ (FermiLab) data on the pion decay into $e^{+} e^{-}$pair. In order to fix the transition form factors in the most model independent way we used corresponding data from CLEO [29] and L3 [30-32] collaborations for the PS and AV mesons transition form factors, respectively (see for details $[13,14]$. We would like to point out, that one can expect the important contribution of the AV exchange to spin dependent part of muon-proton interaction because the exchange particle has the spin one. Furthermore, it is also well known that in the channel with quantum number $1^{++}$axial anomaly effects can play an important role and, in particularly, these effects might be considered as a cornerstone to solve so-called "proton spin crisis" [33, 34]. The other phenomenological input, the meson-nucleon couplings, were determined by using the Regge approach analysis of the deep-inelastic scattering, $f_{1}$ and $a_{1}$ trajectories contributing to the polarization of quarks in the nucleon $[35,36]$.

Calculating the matrix elements with wave functions of $1 \mathrm{~S}, 2 \mathrm{~S}$ and $2 \mathrm{P}_{1 / 2}$ states, we obtain the corresponding contributions to the HFS spectrum

$$
\begin{aligned}
& \Delta E_{\pi}^{h f s}(1 S)=\frac{\mu^{3} \alpha^{5} g_{A}}{6 F_{\pi}^{2} \pi^{3}}\left\{\mathcal{A}(0) \frac{4 W\left(1+\frac{W}{m_{\pi}}\right)}{m_{\pi}\left(1+\frac{2 W}{m_{\pi}}\right)^{2}}-\frac{1}{\pi} \int_{0}^{\infty} \frac{d s}{s} \operatorname{Im} \mathcal{A}(s) \times\right. \\
& \left.\left[1+\frac{1}{4 W^{2}\left(s-m_{\pi}^{2}\right)}\left(\frac{m_{\pi}^{4}}{\left(1+\frac{m_{\pi}}{2 W}\right)^{2}}-\frac{s^{2}}{\left(1+\frac{\sqrt{s}}{2 W}\right)^{2}}\right)\right]\right\}, \\
& \Delta E_{\pi}^{h f s}(2 S)=\frac{\mu^{3} \alpha^{5} g_{A}}{48 F_{\pi}^{2} \pi^{3}}\left\{\mathcal{A}(0) \frac{W\left(8+11 \frac{W}{m_{\pi}}+8 \frac{W^{2}}{m_{\pi}^{2}}+2 \frac{W^{3}}{m_{\pi}^{3}}\right)}{2 m_{\pi}\left(1+\frac{W}{m_{\pi}}\right)^{4}}-\frac{1}{\pi} \int_{0}^{\infty} \frac{d s}{s} \operatorname{Im} \mathcal{A}(s) \times\right. \\
& \left.\left[1+\frac{1}{\left(s-m_{\pi}^{2}\right)}\left(\frac{m_{\pi}^{2}\left(2+\frac{W^{2}}{m_{\pi}^{2}}\right)}{2\left(1+\frac{W}{m_{\pi}}\right)^{4}}-\frac{s\left(2+\frac{W^{2}}{s}\right)}{2\left(1+\frac{W}{\sqrt{s}}\right)^{4}}\right)\right]\right\}, \\
& \Delta E_{A V}^{h f s}(1 S)=\frac{32 \alpha^{5} \mu^{3} g_{A V P P} F_{A V \gamma^{*} \gamma^{*}}^{(0)}(0,0,0)}{3 M_{A}^{2} \pi^{3}\left(1+\frac{2 W}{M_{A}}\right)^{2}} I\left(\frac{m_{\mu}}{\Lambda_{A}}\right) \text {, } \\
& \Delta E_{A V}^{h f s}(2 S)=\frac{2 \alpha^{5} \mu^{3} g_{A V P P} F_{A V \gamma^{*} \gamma^{*}}^{(0)}(0,0,0)\left(2+\frac{W^{2}}{M_{A}^{2}}\right)}{3 M_{A}^{2} \pi^{3}\left(1+\frac{W}{M_{A}}\right)^{4}} I\left(\frac{m_{\mu}}{\Lambda_{A}}\right),
\end{aligned}
$$

where $W=m_{r} \alpha$ and $m_{r}$ is reduced mass.

In Table 1 our results for contribution of the pion and AV mesons exchanges to HFS are presented. For the case of $2 S$ state the total contribution from pion and AV meson exchanges 
Table 1. Pion and axial-vector mesons exchanges contribution to HFS of muonic hydrogen.

\begin{tabular}{|c|c|c|c|c|c|}
\hline mesons & $I^{G}\left(J^{P C}\right)$ & $\begin{array}{c}\Lambda_{A} \\
\text { in } \mathrm{MeV}\end{array}$ & $\begin{array}{c}F_{A V \gamma^{*} \gamma^{*}}^{(0)}(0,0) \\
\text { in } G e V^{-2}\end{array}$ & $\begin{array}{c}\Delta E^{h f s}(1 S) \\
\text { in meV }\end{array}$ & $\begin{array}{c}\Delta E^{h f s}(2 S) \\
\text { in meV }\end{array}$ \\
\hline$f_{1}(1285)$ & $0^{+}\left(1^{++}\right)$ & 1040 & 0.266 & $-0.0093 \pm 0.0033$ & $-0.0012 \pm 0.0004$ \\
\hline$a_{1}(1260)$ & $1^{-}\left(1^{++}\right)$ & 1040 & 0.591 & $-0.0437 \pm 0.0175$ & $-0.0055 \pm 0.0022$ \\
\hline$f_{1}(1420)$ & $0^{+}\left(1^{++}\right)$ & 926 & 0.193 & $-0.0013 \pm 0.0008$ & $-0.0002 \pm 0.0001$ \\
\hline$\pi^{0}$ & $1^{-}\left(0^{-+}\right)$ & 776 & & $-0.0017 \pm 0.0001$ & $-0.0002 \pm 0.00002$ \\
\hline \hline Sum & & & & $-0.0560 \pm 0.0178$ & $-0.0071 \pm 0.0024$ \\
\hline \hline
\end{tabular}

is equal to (-0.0071) $\mathrm{meV}$, which is quite important to obtain the total value of HFS with high precision.

We extend our calculation of muon-proton interaction to the case of scalar mesons. There are several scalar mesons with the mass near $1 \mathrm{GeV}$, which can contribute to the effective muon-proton interaction: $\sigma(550), f_{0}(980), a_{0}(980), f_{0}(1370)$. The general parameterization of scalar meson - two photon vertex function is the following:

$$
\begin{aligned}
& T_{S}^{\mu v}\left(p, k_{1}, k_{2}\right)=e^{2}\left\{A\left(p^{2}, k_{1}^{2}, k_{2}^{2}\right)\left(g^{\mu v}\left(k_{1} \cdot k_{2}\right)-k_{1}^{v} k_{2}^{\mu}\right)+\right. \\
& \left.B\left(p^{2}, k_{1}^{2}, k_{2}^{2}\right)\left(k_{2}^{\mu} k_{1}^{2}-k_{1}^{\mu}\left(k_{1} \cdot k_{2}\right)\right)\left(k_{1}^{v} k_{2}^{2}-k_{2}^{v}\left(k_{1} \cdot k_{2}\right)\right)\right\} .
\end{aligned}
$$

The muon-proton interaction amplitude via the meson exchange has the form:

$$
\begin{aligned}
i \mathcal{M}= & -\frac{\alpha^{2} g_{s}}{\pi^{2}} \int \frac{d^{4} k}{k^{4}\left(k^{2}-2 m_{1} k_{0}\right)} A\left(t^{2}, k^{2}, k^{2}\right)\left(g^{\mu \nu}\left(k_{1} \cdot k_{2}\right)-k_{1}^{v} k_{2}^{\mu}\right) \times \\
& {\left[\bar{u}\left(q_{1}\right) \gamma_{\mu}\left(\hat{p}_{1}-\hat{k}+m_{1}\right) \gamma_{\nu} u\left(p_{1}\right)\right]\left[\bar{v}\left(p_{2}\right) v\left(q_{2}\right)\right] \frac{1}{\mathbf{t}^{2}+M_{s}^{2}}, }
\end{aligned}
$$

where $g_{s}$ is the coupling constant of scalar meson with the proton. We set further in the numerator $t=0$. This leads to the cancelation of the term with the function $B\left(p^{2}, k_{1}^{2}, k_{2}^{2}\right)$. Using again projection operator on muon-proton states with spin $S=0, S=1$ we can construct the interaction operator for these states.

Our analysis shows that there is no contribution of the scalar mesons to HFS in the leading order in $\alpha$. At the same time there are shifts of the levels $2 \mathrm{~S}, 2 \mathrm{P}$ as whole which are determined by the equations:

$$
\begin{gathered}
\Delta E_{\sigma}^{L s}(2 S)=-\frac{\alpha^{5} \mu^{3} g_{s} m_{1} A_{\sigma}}{384 \pi M_{s}^{2}} \frac{\left(2+\frac{W^{2}}{M_{S}^{2}}\right)}{\left(1+\frac{W}{M_{S}}\right)^{4}}\left[-63+180 \ln 2+4 a_{1}^{2}(-20+33 \ln 2)-12\left(15+11 a_{1}^{2}\right) \ln a_{1}\right], \\
\Delta E_{\sigma}^{L s}(2 P)=\frac{\alpha^{5} \mu^{3} g_{s} m_{1} A_{\sigma}}{144 \pi M_{s}^{2}} \frac{\left(3+8 \frac{W}{M_{s}}+\frac{7 W^{2}}{2 M_{s}^{2}}\right)}{\left(1+\frac{W}{M_{s}}\right)^{4}} \\
{\left[-9+36 \ln 2+2 a_{1}^{2}(-7+12 \ln 2)-12\left(3+2 a_{1}^{2}\right) \ln a_{1}\right]}
\end{gathered}
$$

where $a_{1}=2 m_{\mu} / \Lambda$, and the parameterization of function $A\left(t^{2}, k^{2}, k^{2}\right)$ for scalar mesons chosen in the same form as for pseudoscalar and axial-vector mesons (monopole form for variables $k_{1}$ and $k_{2}$ ):

$$
A\left(t^{2}, k^{2}, k^{2}\right)=A_{\sigma} \frac{\Lambda^{4}}{\left(\Lambda^{2}-k^{2}\right)^{2}} .
$$


Coupling constant $A_{\sigma}$ can be related with the decay width $\Gamma_{\sigma \rightarrow \gamma \gamma}$ [41, 42]. Two expressions (23), (24) give contributions which are different in sign just as in the case of pseudoscalar meson. Preliminary numerical estimate of the contribution of $\sigma$-meson to the Lamb shift (2P-2S) is shown in Fig. 1(Right). It was obtained taking the values of parameters for $\sigma$ meson from [43].

\section{Conclusion}

A new important contributions to the muon-nucleon interaction are found. It comes from pion and AV mesons exchanges induced by anomalous meson-two photon vertices. The contribution of this exchange to the HFS of $\mu p$ is calculated in framework of the quasipotential method in QED and the use of the technique of projection operators on the states of two particles with a definite spin. It is shown that this contribution is rather large and should be taken into account for the interpretation of the new data on HFS in this atom. More recently, we studied [18] the contribution of some two-photon interactions to the muon and proton interaction potential and into the fine and hyperfine structure of the energy spectrum for $\mathrm{P}$ states.

As has been mentioned the CREMA Collaboration measured two transition frequencies in muonic hydrogen for the $2 \mathrm{~S}$ triplet state $\left(2 P_{3 / 2}^{F=2}-2 S_{1 / 2}^{F=1}\right)$ and for $2 \mathrm{~S}$ singlet state $\left(2 P_{3 / 2}^{F=1}-\right.$ $2 S_{1 / 2}^{F=0}$ ) [2]. From these measurements it is possible to extract the value of HFS for $2 S$ level. Obtained value (4) allows to get the value of the Zemach radius (8) with accuracy $3.4 \%$ with help of relation (6). This is in the agreement with another numerical values $r_{Z}=1.086(12) \mathrm{fm}$ [37], $r_{Z}=1.045(4) \mathrm{fm}$ [38], $r_{Z}=1.047(16) \mathrm{fm}$ [39], $r_{Z}=1.037(16) \mathrm{fm}$ [40] obtained from electron-proton scattering and from $\mathrm{H}$ and muonium spectroscopy. We should emphasize that the changing of the theoretical value of HFS on $0.001 \mathrm{meV}$ leads to the changing of the Zemach radius on $0.006 \mathrm{fm}$. Therefore, our contribution coming from the pion and AV meson exchanges leads to the new value of this radius

$$
r_{Z}=1.040(37) \mathrm{fm},
$$

which is smaller in the comparison with most listed results but still agree with them within errorbars.

The work is supported by Russian Science Foundation (grant No. RSF 18-12-00128) and Russian Foundation for Basic Research (grant No. 18-32-00023) (F.A.M.).

\section{References}

[1] R. Pohl, A. Antognini, F. Nez et al., Nature 466, 213 (2010).

[2] A. Antognini et al., Science 339, 417 (2013).

[3] A. Antognini et al., Ann. Phys. (NY) 331, 127 (2013).

[4] A. E. Dorokhov, A. E. Radzhabov, A. S. Zhevlakov, Eur. Phys. J. C 72, 2227 (2012).

[5] A. E. Dorokhov, A. E. Radzhabov, A. S. Zhevlakov, JETP Lett. 100, 133 (2014).

[6] R. Pohl, F. Nez, L. M. P. Fernandes et al., Science 353, 669 (2016).

[7] P. J. Mohr, D. B. Newell, and B. N. Taylor (CODATA Recommended Values of the

Fundamental Physical Constants: 2014) Rev. Mod. Phys. 88, 035009 (2016).

[8] Y. Ma et al., Int. J. Mod. Phys. Conf. Ser. 40, 1660046 (2016).

[9] A. Adamczak et al. [FAMU Collaboration], JINST 11, no. 05, P05007 (2016).

[10] R. Pohl [CREMA Collaboration], J. Phys. Soc. Jap. 85, 091003 (2016).

[11] A. Beyer, et al., Science 358, 79 (2017). 
[12] H. Fleurbaey et al., Phys. Rev. Lett. 120, 183001 (2018).

[13] A. E. Dorokhov, N. I. Kochelev, A. P. Martynenko, F. A. Martynenko, and R. N. Faustov, Phys. Part. Nucl. Lett. 14, 857 (2017) ; arXiv:1704.07702 [hep-ph].

[14] A. E. Dorokhov, N. I. Kochelev, A. P. Martynenko, F. A. Martynenko, and

A. E. Radzhabov, Phys. Lett. B 776, 105 (2018).

[15] H. Q. Zhou, H. R. Pang, Phys. Rev. A 92, 032512 (2015).

[16] F. Hagelstein, V. Pascalutsa, PoS CD15 077 (2016).

[17] N. T. Huong, E. Kou, B. Moussallam, Phys. Rev. D 93, 114005 (2016).

[18] A. E. Dorokhov, N. I. Kochelev, A. P. Martynenko, F. A. Martynenko and A. E. Radzhabov, Eur. Phys. J. A 54, 131 (2018); arXiv:1804.09749 [hep-ph].

[19] A. P. Martynenko and R. N. Faustov, J. Exp. Theor. Phys. 98, 39 (2004).

[20] A. P. Martynenko and R.N. Faustov, J. Exp. Theor. Phys. 88, 672 (1999).

[21] A. A. Krutov, A. P. Martynenko, F. A. Martynenko and O. S. Sukhorukova, Phys. Rev. A 94, 062505 (2016).

[22] M. I. Eides, H. Grotch, and V. A. Shelyuto, Theory of Light Hydrogenic Bound States, Springer Tracts in Modern Physics, V. 222 (Springer, Berlin, Heidelbeg, New York, 2007).

[23] K. Pachucki, Phys. Rev. A 53, 2092 (1996).

[24] E. Borie, Ann. Phys. 327, 733 (2012).

[25] R. N. Faustov and A. P. Martynenko, Phys. Lett. B 541, 135 (2002).

[26] A. P. Martynenko, F. A. Martynenko, and R. N. Faustov, J. Exp. Theor. Phys. 124, 895 (2017).

[27] A. E. Dorokhov and M. A. Ivanov, Phys. Rev. D 75, 114007 (2007).

[28] A. E. Dorokhov, M. A. Ivanov and S. G. Kovalenko, Phys. Lett. B 677, 145 (2009).

[29] J. Gronberg et al. [CLEO], Phys. Rev. D57, 33 (1998).

[30] P. Achard et al. [L3 Collaboration], Phys. Lett. B 526, 269 (2002).

[31] P. Achard et al. [L3 Collaboration], JHEP 03, 018 (2007).

[32] H. Aihara et al., Phys. Rev. D 38, 1 (1988).

[33] A.E. Dorokhov, N.I. Kochelev and Yu.A. Zubov, Int. J. Mod. Phys. A 8 (1993) 603.

[34] M. Anselmino, A. Efremov and E. Leader, Phys. Rept. 261, 1 (1995); Erratum: [Phys. Rept. 281, 399 (1997)]

[35] N. I. Kochelev and Y. Oh, Phys. Rev. D 85, 016012 (2012).

[36] N. I. Kochelev, D.-P. Min, Y. Oh, V. Vento, and A. V. Vinnikov, Phys. Rev. D 61, 094008 (2000).

[37] J. Friar and I. Sick, Phys. Lett. B 579, 285 (2004).

[38] M. O. Distler, J. C. Bernauer, and T. Walcher, Phys. Lett. B 696, 343 (2011).

[39] A. V. Volotka, V. M. Shabaev, G. Plunien, and G. Soff, Eur. Phys. J. D 33, 23 (2005).

[40] A. Dupays, A. Beswick, B. Lepetit, C. Rizzo, and D. Bakalov, Phys. Rev. A 68, 052503 (2003).

[41] M. K. Volkov, E. A. Kuraev, and Yu. M. Bystritskiy, Phys. Atom. Nucl. 73, 443 (2010).

[42] F Giacosa, Th. Gutsche, and V. E. Lyubovitskij, Phys. Rev. D 77, 034007 (2008).

[43] H.-Q. Zhou, Phys. Rev. C 95, 025203 (2017). 